\title{
Capsosomes as Long-Term Delivery Vehicles for
}

\section{Protein Therapeutics}

James W. Maina, Joseph J. Richardson, ${ }^{\S}$ Rona Chandrawati, ${ }^{\dagger}$ Kristian Kempe, ${ }^{\ddagger}$ Martin P. van Koeverden, and Frank Caruso*

ARC Centre of Excellence in Convergent Bio-Nano Science and Technology, and the

Department of Chemical and Biomolecular Engineering, The University of Melbourne, Parkville, Victoria 3010, Australia

KEYWORDS: capsosomes, liposomes, proteins, layer-by-layer

\section{ABSTRACT}

We report the preparation of polymer capsules containing liposomal subcompartments, termed capsosomes, and their ability for the sustained delivery of protein therapeutics. Capsosomes were formed through the layer-by-layer (LbL) assembly of polymers and protein-loaded liposomes, followed by the formation of a capsule membrane based on disulfide cross-linked poly(methacrylic acid). The loading capacities of a model cargo (lysozyme), and brain-derived neurotrophic factor (BDNF), an important neurotrophin that has significant physiological functions on the nervous system, were determined, and the longterm release kinetics of the proteins was investigated in simulated physiological conditions. The capsosomes exhibited protein loading and release behavior that can be tuned by the lipid 
composition of the liposomal compartments, where inclusion of anionic lipids resulted in enhanced protein loading and slower release over the course of 80 days. These findings highlight the potential of capsosomes in the long-term delivery of protein therapeutics.

\section{INTRODUCTION}

With advancements in biotechnology, the number of protein pharmaceutics have dramatically increased, improving the treatment of previously hard-to-treat diseases. ${ }^{1}$ Compared to synthetic small molecules, protein therapeutics have a significant advantage due to their specific mechanism of action and high potency. ${ }^{1,2}$ However, the poor pharmacokinetics of protein therapeutics and their susceptibility to degradation present clinical challenges for their successful delivery. ${ }^{3}$ For example, oral administration can result in digestion of the protein in the gastrointestinal tract, while intravenous injection can result in degradation of the protein by proteases in the blood or in capture of the protein by receptors and non-specific binding sites. ${ }^{4}$ As a result, different delivery approaches have been developed to circumvent the challenges related to protein delivery.

To date, the two most prominent techniques for improving the delivery of protein therapeutics are PEGylation ${ }^{5,6}$ and incorporation of the proteins into biocompatible carriers. ${ }^{7,8}$ PEGylation, the chemical attachment of poly(ethylene glycol) (PEG) to proteins, can enhance overall protein efficacy by masking antigenic sites, thus reducing the formation of neutralizing antibodies. ${ }^{2}$ The PEG shell also protects the proteins from proteolytic degradation by proteases present in the bloodstream. Finally, it improves the solubility and increases the hydrodynamic radius of the proteins, which reduces glomerular filtration. The major drawback of PEGylation, however, is the reduction in protein activity that commonly follows chemical modification of the protein. ${ }^{2}$ In addition, PEG is not biodegradable and 
long-term usage may lead to accumulation in the liver, which could increase the risk of toxicity. ${ }^{8}$

Alternatively, a wide variety of carriers have also been evaluated for protein delivery, including polymeric particles, hydrogels, and liposomes. ${ }^{2,49}$ Poly(lactic-co-glycolic acid) (PLGA)-based systems are among the most successful polymeric carriers, with numerous iterations, such as Lupron depot, Nutropin depot and Decapeptyl, being already available on the market. ${ }^{1,7}$ The success of PLGA stems from its high biocompatibility and biodegradability. ${ }^{7}$ The physical properties and biodegradability of PLGA can also be tuned to fit specific applications by varying the molecular weight and the ratio of lactic acid to glycolic acid. ${ }^{10}$ However, PLGA still suffers from some drawbacks, such as hydrophobicity, an acidic microenvironment during degradation, and chemical reactivity with some protein therapeutics. ${ }^{1}$

Liposomes are a class of biologically inspired carriers that have potential in the delivery of protein therapeutics. Compared to other carriers, liposomes have significant advantages due to their simplicity, versatility and biological nature. ${ }^{11}$ Liposome properties can be easily controlled by manipulating the phospholipid compositions, which has made liposomes the most prevalent carriers in clinical applications in the delivery of a wide variety of therapeutics, including doxorubicin, cisplatin, and annamycin. ${ }^{12,13}$ Their hydrophilic core provides a confined microenvironment for labile protein therapeutics, while the hydrophobic lipid bilayer membrane insulates the encapsulated cargo from potential degradative environments. $^{12,13}$ Still, the fragile mechanical properties and in vivo instability of bare liposomes $^{14}$ limit their application in long-term delivery, which is necessary for a range of protein therapeutics. ${ }^{15,16}$

We recently demonstrated the stable incorporation of intact liposomes into polymer capsules to form capsosomes. This represents a multicompartmentalization approach for 
enhancing the physical integrity of liposomes, while preserving their unique biomedical properties. ${ }^{17-19}$ Multilayers of liposomes composed of zwitterionic or negatively charged, unsaturated or saturated phospholipids can be incorporated into polymer films, allowing the assembly of capsosomes with more than 100000 liposomal compartments, ${ }^{19}$ thus enabling high loadings of therapeutic cargo. Enzymatic proteins can be efficiently encapsulated in the aqueous environment of the liposomal compartments of the capsosomes without the need for chemical modification. Furthermore, their biological activity can be more effectively retained compared to proteins directly encapsulated in bare polymer capsules without liposomal compartments. ${ }^{18,19}$ These properties highlight the potential of capsosomes as delivery carriers for protein therapeutics.

Herein, we demonstrate the application of capsosomes in the sustained delivery of protein therapeutics. The study uses lysozyme as a model protein and brain-derived neurotrophic factor (BDNF), a neurotrophin that has been shown to be one of the most potent in the treatment of neurodegenerative diseases. ${ }^{20,21}$ Animal studies have demonstrated the therapeutic efficacy of BDNF in the treatment of Alzheimer’s, ${ }^{22}$ and Parkinson's; ${ }^{23}$ however, our target application is delivery to the inner ear for neurodegenerative hearing loss due to cochlear implantation. ${ }^{24}$ In this context the capsosomes would be injected into the inner ear during surgery to allow for the optimum therapeutic effect of BDNF, which is sustained release for over 30 days. In this study, we (i) assemble capsosomes containing multilayers of protein-loaded liposomes; (ii) evaluate the protein loading capacity for liposomal compartments based on different lipid compositions; and (iii) investigate the long-term release profile of the protein therapeutics in simulated physiological conditions. Capsosomes were prepared using the layer-by-layer (LbL) assembly of polymers and protein-loaded liposomes, followed by the formation of disulfide cross-linked poly(methacrylic acid) carrier capsules, as previously reported. ${ }^{18,19}$ The influence of liposomal composition was 
investigated by evaluating different liposomes made of zwitterionic lipids, 1,2-dimyristoylsn-glycero-3-phosphocholine (DMPC) and 1,2-dipalmitoyl-sn-glycero-3-phosphocholine (DPPC), as well as an anionic lipid, 1,2-dioleoyl-sn-glycero-3-phospho-L-serine (DOPS).

\section{EXPERIMENTAL}

Materials. 1,2-dimyristoyl-sn-glycero-3-phosphocholine (DMPC), 1,2-dipalmitoyl-snglycero-3-phosphocholine (DPPC), and 1,2-dioleoyl-sn-glycero-3-phospho-L-serine (DOPS) were obtained from Avanti Polar Lipids, USA. Chloroform, poly(L-lysine) (PLL, $\left.M_{w}=40-60 \mathrm{kDa}\right), \operatorname{poly}\left(N\right.$-vinyl pyrrolidone) (PVPON, $\left.M_{w}=10 \mathrm{kDa}\right)$, fluorescein isothiocyanate (FITC), 4-(2-hydroxyethyl)piperazine-1-ethanesulfonic acid (HEPES), sodium acetate (NaOAc), 2-( $\mathrm{N}$-morpholino)ethanesulfonic acid (MES), chloramine-T hydrate, and DL-dithiothreitol (DTT) were purchased from Sigma-Aldrich. Brain-derived neurotrophic factor (BDNF) was obtained from GenWay Biotech Inc. Silica templates (2.59 $\mu \mathrm{m}$ in diameter) were purchased from Microparticles GmbH, Germany. Poly[(methacrylic acid)-co(cholesteryl methacrylate)] ( $\mathrm{PMA}_{c}, M_{w}=18 \mathrm{kDa}, 13$ mol\% cholesteryl methacrylate) and thiol-functionalized poly(methacrylic acid) $\left(\mathrm{PMA}_{\mathrm{SH}}\right)$ with $14 \mathrm{~mol} \%$ thiol modification were synthesized following previously reported protocols. ${ }^{19}$

Protein Labeling. $180 \mu \mathrm{L}$ of FITC $\left(1 \mathrm{mg} \mathrm{mL}^{-1}\right.$ in DMSO) was added to $6 \mathrm{~mL}$ of $1 \mathrm{mg} \mathrm{mL} \mathrm{m}^{-1}$ lysozyme in PBS buffer ( $\mathrm{pH}$ 8) under vigorous vortexing. The sample was then protected from light and reacted for $2 \mathrm{~h}$ at room temperature $\left(25^{\circ} \mathrm{C}\right)$ with mixing. Subsequently, the sample was purified by centrifugation at $14000 \mathrm{~g}$ for $20 \mathrm{~min}$ using a Nanosep spin column (MWCO $=3 \mathrm{kDa}$ ) to remove the unreacted dye. The labeled lysozyme was then redispersed in $3 \mathrm{~mL}$ of Milli-Q water and the protein concentration was determined 
using a NanoDrop 1000 UV/VIS spectrophotometer. The same protocol was followed for labeling the BDNF with a F/P ratio of approximately 1.

Liposome Formation. Liposomes were prepared by a thin film hydration technique using various lipid formulations of zwitterionic DMPC and DPPC, and anionic DOPS. Briefly, lipids (2.5 mg) were dissolved in chloroform at $50 \mathrm{mg} \mathrm{mL}^{-1}$ in a round-bottomed flask, and dried for $1 \mathrm{~h}$ under nitrogen flow to form a thin lipid film on the sides of the flask. The film was then hydrated with $1 \mathrm{~mL}$ of $30 \mu \mathrm{g} \mathrm{mL}^{-1}$ lysozyme-FITC or $20 \mu \mathrm{g} \mathrm{mL}{ }^{-1}$ BDNF-FITC in HEPES buffer (10 mM HEPES, $150 \mathrm{mM} \mathrm{NaCl,} \mathrm{pH} \mathrm{7.4)} \mathrm{to} \mathrm{form} \mathrm{multilamellar} \mathrm{liposomes.} \mathrm{The}$ liposomes were then extruded 31 times through $100 \mathrm{~nm}$ filters, resulting in unilamellar liposomes. $\zeta$-potentials of liposomes prepared at various lipid compositions are presented in Table S1.

Assembly of Multilayer Capsosomes. Capsosomes were assembled using a previously reported procedure. ${ }^{19}$ Briefly, $5 \mathrm{mg}$ of silica templates $(2.59 \mu \mathrm{m}$ in diameter) were washed three times (1000 $\mathrm{g}$ for $30 \mathrm{~s}$ ) using HEPES buffer. The particles were then suspended in 100 $\mu \mathrm{L}$ of PLL (1 $\mathrm{mg} \mathrm{mL}^{-1}$ ) and incubated for 15 min to allow PLL to form a precursor layer on the silica templates. The PLL-coated particles were then washed three times with HEPES buffer. Lysozyme- or BDNF-loaded liposomes $(100 \mu \mathrm{L})$ was added to the particles, vortexed for $30 \mathrm{~s}$ and incubated for $45 \mathrm{~min}$. The particles were then washed three times to remove excess liposomes, followed by the adsorption of a PMA (for zwitterionic liposomes) or $\mathrm{PMA}_{\mathrm{c}} / \mathrm{PLL}$ (for negatively charged liposomes) separation layer (15 min) and washed three times with HEPES buffer. After the adsorption of five liposome layers, a capping layer of $\mathrm{PMA}_{\mathrm{c}}$ was adsorbed and the buffer was exchanged to NaOAc buffer (20 mM NaOAc, pH 4). Seven alternating layers of PVPON and PMA $\mathrm{SH}\left(1 \mathrm{mg} \mathrm{mL} \mathrm{m}^{-1}\right)$ were adsorbed followed by cross-linking of the thiol groups within the polymer layers by the addition of $400 \mu \mathrm{L}$ of chloramine-T (2.5 mM) in MES buffer (50 mM MES, pH 6) under vigorous vortexing for 1 
min at room temperature $\left(25^{\circ} \mathrm{C}\right)$. Finally, the silica core was removed by buffered hydrofluoric acid (HF) (2 M HF and $8 \mathrm{M} \mathrm{NH}_{4} \mathrm{~F}$ ) [Caution! HF is extremely toxic and corrosive! Handle with care], followed by washing three times (4500 $\mathrm{g}$ for $3 \mathrm{~min}$ ) with NaOAc buffer.

Protein Quantification. To quantify the amount of protein loaded in the capsosomes, 10 $\mu \mathrm{L}$ of each capsosome sample was mixed with $2 \mu \mathrm{L}$ of $0.5 \mathrm{M}$ DTT, a reducing agent that cleaves disulfide bonds within the polymer capsules, and incubated at $37^{\circ} \mathrm{C}$ for $30 \mathrm{~min} .2 \mu \mathrm{L}$ of Triton-X (10\% v/v) was then added to disrupt the liposome compartments and the sample was incubated for a further 15 min with gentle mixing. This was followed by sonication for 3 min at $41{ }^{\circ} \mathrm{C}$, after which the sample was diluted in PBS buffer to a total volume of $450 \mu \mathrm{L}$. The fluorescence intensity of the sample was then measured at an excitation wavelength of $488 \mathrm{~nm}$ and at an emission wavelength of $513 \mathrm{~nm}$. The amount of encapsulated protein was quantified by correlation with fluorescence calibration curves of lysozyme-FITC or BDNFFITC in PBS buffer (Figures S1 and S2).

Protein Release from Capsosomes. The in vitro release was studied by incubating capsosomes, corresponding to approximately $0.55 \mu$ g lysozyme or $1.5 \mu \mathrm{g}$ BDNF, in $100 \mu \mathrm{L}$ PBS buffer at $37{ }^{\circ} \mathrm{C}$ and $4{ }^{\circ} \mathrm{C}$. At various time intervals, the samples were centrifuged at $4500 \mathrm{~g}$ for $3 \mathrm{~min}$ and $80 \mu \mathrm{L}$ of the supernatant was withdrawn to assess the amount of protein released. The samples were then refilled with fresh buffer and gently vortexed before incubation for further release. The amount of protein released was quantified by measuring the fluorescence intensity at an excitation wavelength of $488 \mathrm{~nm}$ and at an emission wavelength of $513 \mathrm{~nm}$, and correlated to the calibration curves. The percentage of protein released was calculated as follows: release $(\%)=$ (cumulative protein release $) /($ total protein loaded) $\times 100 \%$. 
Instrumentation. An inverted Olympus IX71 microscope equipped with a $60 \times$ oil immersion objective lens (Olympus UPFL20/0.5 NA, W.D. 1.6) and a CCD camera mounted on the left-hand port of the microscope was used to acquire the fluorescence microscopy images. The concentration of the fluorescently labeled protein was measured using a NanoDrop 1000 UV/Vis spectrophotometer (Thermo Scientific) by measuring the absorbance at $280 \mathrm{~nm}$, while fluorescence measurements were obtained using a Fluorog-3 spectrofluorometer (Horiba Jobin Yvon). Microelectrophoresis of liposomes prepared at different lipid compositions was performed using a Malvern High Performance Particle Sizer (HPPS), while capsosome counting was undertaken by flow cytometry (Cyflow Space, Partec $\mathrm{GmbH})$.

\section{RESULTS AND DISCUSSION}

\section{Lysozyme-loaded multilayer capsosomes}

The assembly of multilayer capsosomes was performed based on our previously optimized protocol, via the sequential adsorption of polymer and liposome layers onto silica particle templates. ${ }^{19}$ A PLL precursor layer was first adsorbed onto silica particles, followed by the deposition of fluorescently labeled protein-loaded liposomes. PMA ${ }_{c}$ was used as a polymer separation layer to enable consecutive adsorption of zwitterionic saturated liposome layers (Scheme 1). 
Scheme 1. Schematic representation of the assembly of multilayer capsosomes encapsulating protein therapeutics in the liposomal compartments. Silica particle templates are coated with a polymer precursor layer (i), followed by alternating layers of protein-loaded liposomes and polymer separation layers (ii and iii). A polymer capping layer is adsorbed after the deposition of multiple protein-loaded liposome layers, followed by alternating layers of poly( $N$-vinyl pyrrolidone) (PVPON) and thiol-functionalized poly(methacrylic acid) $\left(\mathrm{PMA}_{\mathrm{SH}}\right.$ ) via layer-by-layer (LbL) assembly (iv). Removal of the silica core results in capsosome formation (v).

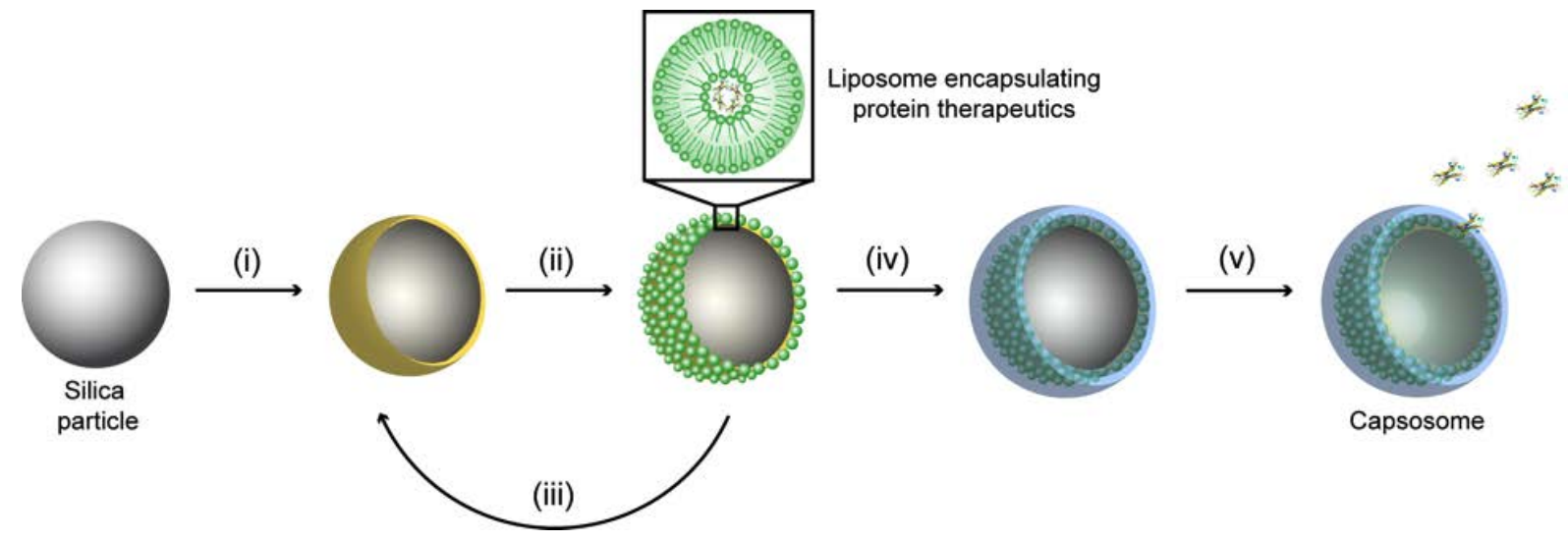

Fluorescently labeled lysozyme was first used as a model protein to investigate the loading capacity in capsosomes and the protein release profile because of its properties to BDNF (lysozyme $M_{w}=14 \mathrm{kDa}$, isoelectric point (pI) 10.5; BDNF $\left.M_{w}=13 \mathrm{kDa}, \mathrm{pI} 10\right) .{ }^{25,26}$ The fluorescence intensity of the particles due to the adsorbed lysozyme-FITC-loaded zwitterionic saturated DMPC/DPPC liposomes was monitored by spectrofluorometry. The consecutive adsorption steps resulted in a linear buildup of protein loading over five liposome deposition steps (Figure 1). This approach allows facile control over the amount of protein encapsulated per individual carrier. 


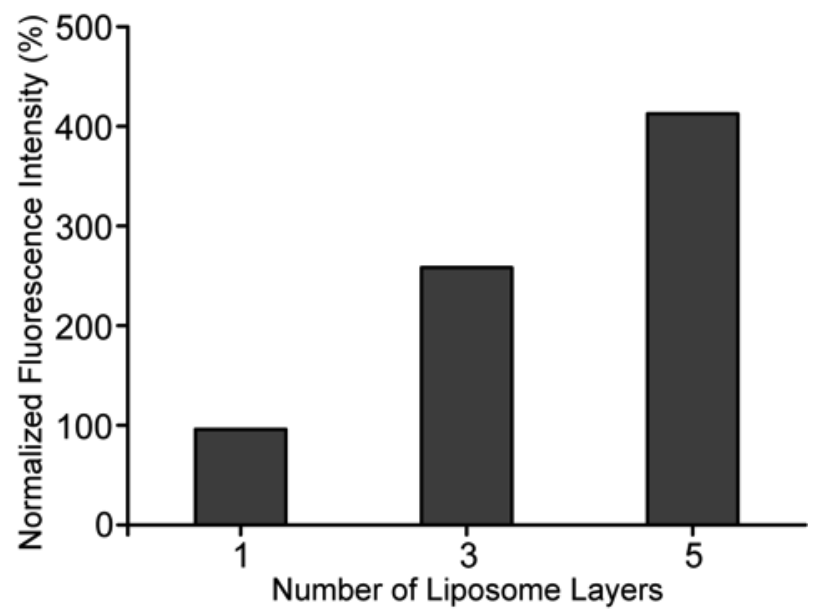

Figure 1. Normalized fluorescence intensity of silica template particles coated with a different number of lysozyme-FITC-loaded zwitterionic DMPC/DPPC liposome layers, as measured by spectrofluorometry.

We examined different lipid compositions of the liposomal compartments that form the capsosomes to assess their effect on protein loading and release. In this study, three different lipid compositions were investigated: (i) zwitterionic DMPC/DPPC, (ii) zwitterionic DPPC, and (iii) negatively charged DMPC/DPPC/DOPS. Lysozyme-FITC was encapsulated in the liposomal compartments and adsorbed onto the polymer-coated particles. After five polymer/liposome bilayers, alternating layers of PVPON and PMA $\mathrm{SH}_{\mathrm{SH}}$ were adsorbed, and the polymer membrane was stabilized by cross-linking of the PMA $\mathrm{SH}_{\mathrm{SH}}$ layers by oxidation of their pendant thiol groups. The amount of encapsulated lysozyme in capsosomes assembled with five liposome layers was compared and quantified by correlation with a fluorescence calibration curve of lysozyme-FITC (Figure S1). The capsosomes exhibited different protein loading behavior depending on the lipid composition of the liposomal compartments (Figure 2). Lysozyme encapsulated in zwitterionic DMPC/DPPC and DPPC liposomes had a loading capacity of $0.020-0.025$ pg of protein per capsosome. On the other hand, the incorporation of negatively charged DOPS resulted in significantly enhanced protein loading, (ca. 0.065 pg of 
lysozyme per capsosome), which is 2.5-fold higher compared with encapsulation in the zwitterionic liposomes (Figure 2a). This high loading may be attributed to the favorable adsorption of the anionic liposomes ( $\zeta$-potential $-12 \pm 1 \mathrm{mV}$, Table S1) to the positively charged PLL film, ${ }^{19}$ as well as the electrostatic interaction between the positively charged proteins at physiological conditions $(\mathrm{pH}$ 7.4) and the negatively charged liposomal membrane. Fluorescence microscopy images confirmed the higher lysozyme loading in capsosomes with five layers of negatively charged DMPC/DPPC/DOPS liposomes in comparison to those with five layers of zwitterionic DMPC/DPPC liposomes (Figure 2b), as evidenced by the difference in brightness between the different types of capsules.
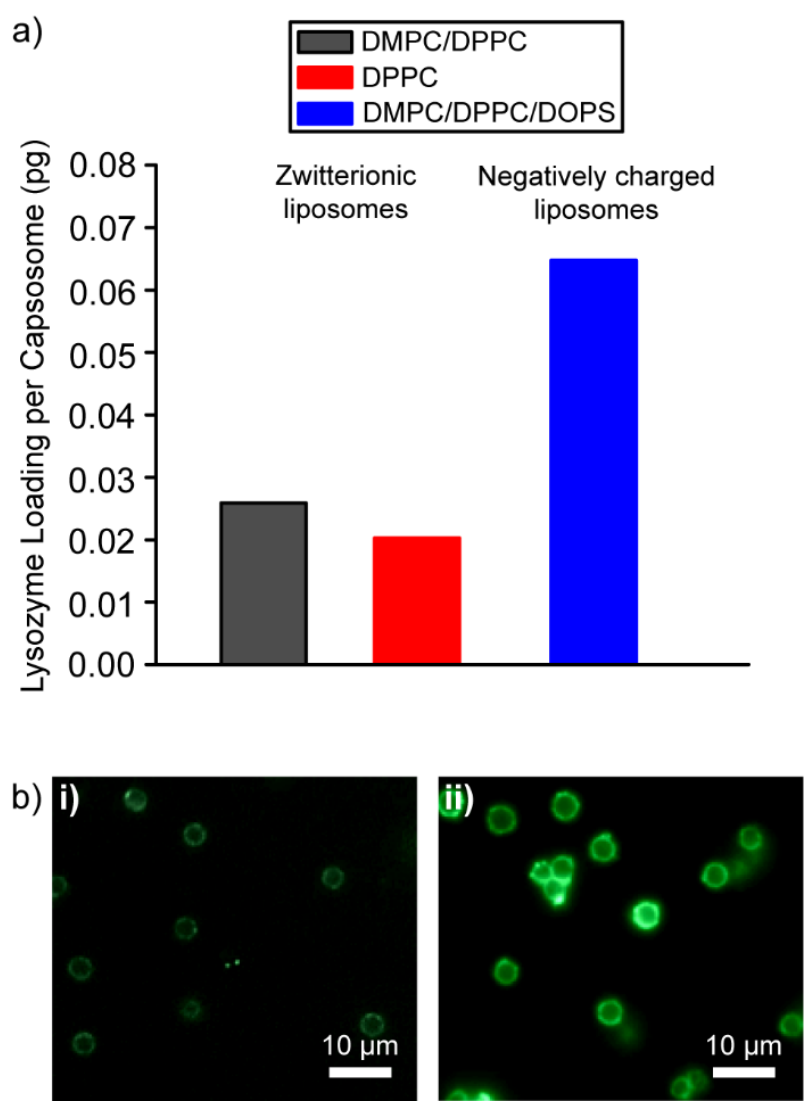

Figure 2. Encapsulation of lysozyme in capsosomes. a) Quantification of protein encapsulated per capsosome with five lysozyme-loaded liposome layers: zwitterionic DMPC/DPPC liposomes (black), zwitterionic DPPC liposomes (red), and negatively charged 
DMPC/DPPC/DOPS liposomes (blue). b) Fluorescence microscopy images of capsosomes with five lysozyme-FITC-loaded liposome layers: zwitterionic DMPC/DPPC liposomes (i) and negatively charged DMPC/DPPC/DOPS liposomes (ii) taken under identical imaging conditions. Incorporation of anionic DOPS phospholipid resulted in enhanced lysozyme loading.

Protein release studies were conducted by incubating the lysozyme-FITC-loaded capsosomes in PBS buffer (pH 7.4) and monitoring the amount of protein in the supernatant over time using spectrofluorometry. Overall, the capsosomes exhibited a two-phase release profile, where rapid release was observed within the first 7 days followed by a sustained release of lysozyme for more than 2 months (Figure 3). An increased content of saturated gel phase lipid (DPPC) resulted in a significant reduction in the initial burst release; the total release of lysozyme over 7 days decreased from ca. 70\% for capsosomes with DMPC/DPPC liposomes to ca. $40 \%$ for capsosomes with DPPC liposomes. This demonstrates that the permeability of liposomes to water soluble cargo can be controlled by varying the ratio of a more rigid lipid bilayer (DPPC) to that of a more fluid lipid bilayer (DMPC). ${ }^{27-29}$ Increasing the content of DPPC, which forms a more rigid membrane as a result of stronger intermolecular forces between the phospholipid bilayers, ${ }^{30}$ results in reduced membrane permeability and a decreased release rate. The inclusion of negatively charged lipid (DOPS) also resulted in a slower release of the lysozyme from the capsosomes with DMPC/DPPC/DOPS liposomes, in part due to the electrostatic interaction with the positively charged proteins. After this time, capsosomes made of the different lipid compositions exhibited similar release rates. These results show that the cargo release profile of capsosomes can be tuned by the lipid composition of the liposome compartments. 


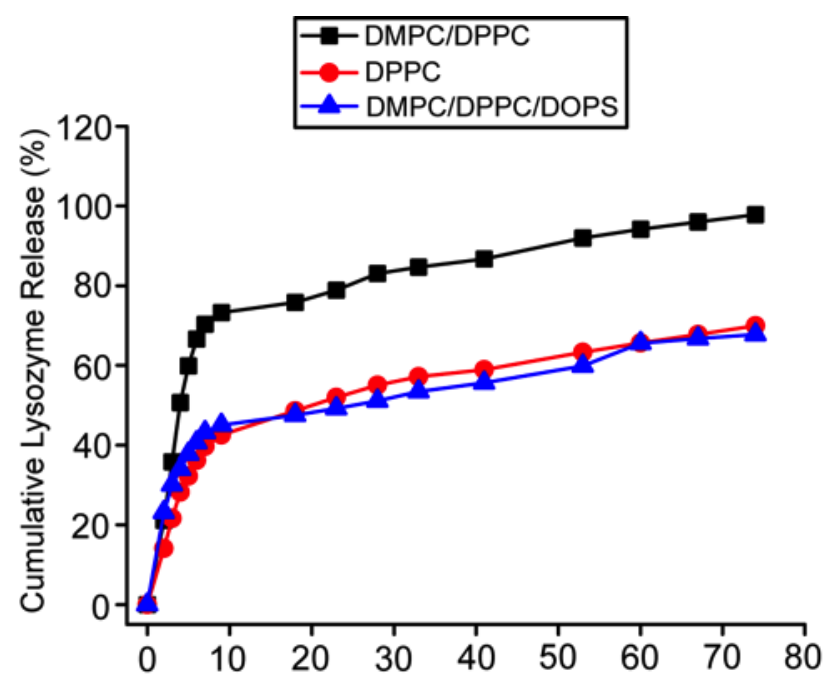

Time (days)

Figure 3. Protein release profiles of lysozyme-loaded capsosomes with five liposome layers in PBS buffer (pH 7.4): zwitterionic DMPC/DPPC liposomes (black), zwitterionic DPPC liposomes (red), and negatively charged DMPC/DPPC/DOPS liposomes (blue).

\section{BDNF-loaded multilayer capsosomes}

After demonstrating the potential of capsosomes as long-term delivery vehicles for protein cargo, we further assessed the loading capacity and release profile of BDNF, a physiologically relevant protein therapeutic, from multilayer capsosomes. In this study, BDNF was fluorescently labeled with FITC and the amount of encapsulated BDNF in capsosomes assembled with five liposome deposition steps was quantified by correlation with a calibration curve of BDNF-FITC (Figure S2). Similarly, the different lipid compositions resulted in a different amount of BDNF encapsulated in the capsosomes (Figure 4). Capsosomes assembled with zwitterionic DMPC/DPPC and DPPC liposomes had a loading capacity of $0.045-0.070 \mathrm{pg}$ BDNF per capsosome, while the addition of negatively charged DOPS in the liposomes resulted in an enhanced loading of 0.12 pg BDNF per capsosome (Figure 4a). This is an appropriate loading capacity, as 4-7 ng of BDNF has been found to be sufficient to elicit a positive therapeutic outcome, indicating that only thousands of 
capsosomes would be required. ${ }^{24}$ However, for the release experiments higher amounts were used to ensure the therapeutic efficacy remained over a prolonged period. In all of the liposome formulations studied, the capsosomes had a relatively higher loading capacity for BDNF in comparison to lysozyme (Figure 2). This is likely due to the relatively larger dimeric molecular structure of $\mathrm{BDNF},{ }^{31}$ an observation consistent with our previous study where capsosomes were observed to exhibit size dependent retention of water soluble cargo. ${ }^{19}$ Fluorescence microscopy images of capsosomes encapsulating BDNF-FITC in the liposomal compartments are shown in Figure 4b.

The release profiles of BDNF from these multilayer capsosomes were investigated at physiological conditions over time, at both $4{ }^{\circ} \mathrm{C}$ and $37^{\circ} \mathrm{C}$. The capsosomes exhibited longterm release behavior, with the total amount of BDNF not fully released after 80 days. The inclusion of anionic DOPS similarly resulted in a reduction in the release rate of the proteins during the first release phase over 7 days (Figure 5). The total release of BDNF from capsosomes with DMPC/DPPC/DOPS liposomes over 7 days was determined to be ca. 30\%, compared with ca. $40 \%$ for capsosomes with DPPC liposomes. The influence of saturated gel phase lipid DPPC in tuning the release profile was well illustrated by comparing the release of BDNF at $37{ }^{\circ} \mathrm{C}$ to those at $4{ }^{\circ} \mathrm{C}$. Over a two-month period, capsosomes with DPPC liposomes released less than $25 \%$ of the encapsulated BDNF at $4{ }^{\circ} \mathrm{C}$, compared with ca. $60 \%$ release at $37^{\circ} \mathrm{C}$. The DPPC lipid bilayers were more tightly packed at $4{ }^{\circ} \mathrm{C}$ than at $37^{\circ} \mathrm{C}$ due to a reduction in thermal vibrations; this resulted in enhanced intermolecular attraction and hence stronger resistance to protein diffusion. ${ }^{32}$ 

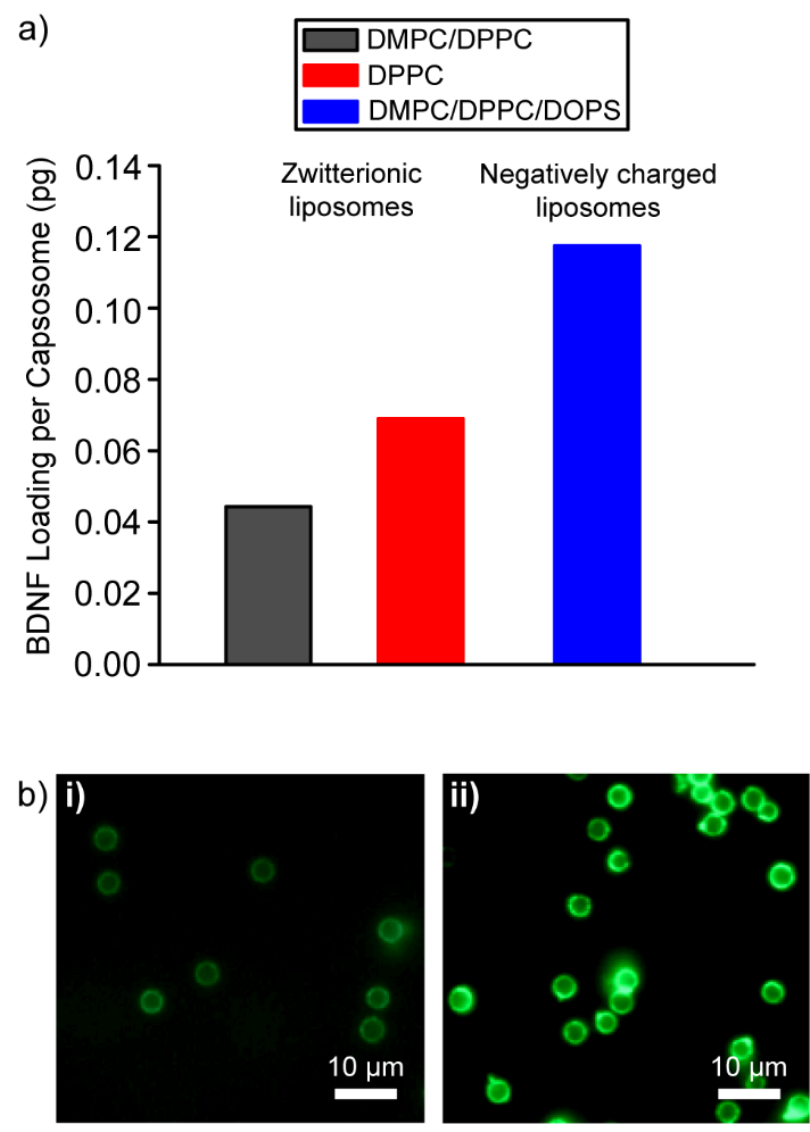

Figure 4. Encapsulation of BDNF in capsosomes. a) Quantification of protein encapsulated per capsosome with five BDNF-loaded liposome layers: zwitterionic DMPC/DPPC liposomes (black), zwitterionic DPPC liposomes (red), and negatively charged DMPC/DPPC/DOPS liposomes (blue). b) Fluorescence microscopy images of capsosomes with five BDNF-FITC-loaded liposome layers: zwitterionic DMPC/DPPC liposomes (i) and negatively charged DMPC/DPPC/DOPS liposomes (ii) taken under identical imaging conditions. Incorporation of anionic DOPS phospholipid resulted in enhanced BDNF loading. 


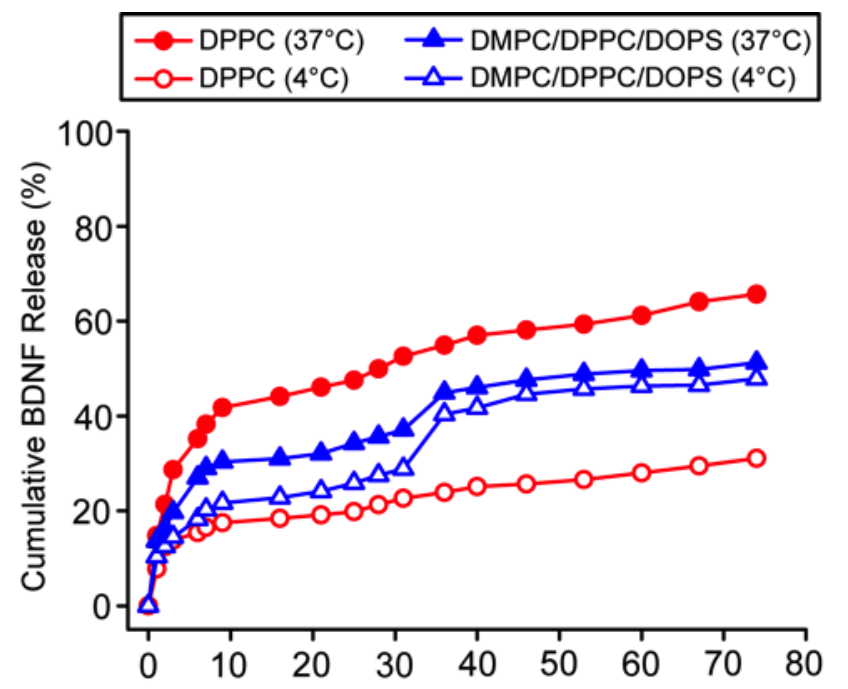

Time (days)

Figure 5. Protein release profiles of BDNF-loaded capsosomes with five liposome layers in PBS buffer (pH 7.4): zwitterionic DPPC liposomes (red) and negatively charged DMPC/DPPC/DOPS liposomes (blue).

Taken together, our results demonstrate that capsosomes can be used as long-term delivery vehicles for protein therapeutics. The versatility of this platform is shown through the ability to tune the amount of protein loaded by tuning the number of deposited liposome layers or liposomal composition. This multicompartmentalization approach allows for the possibility of co-encapsulation of different protein therapeutics, where different release rates of different proteins can be achieved based on different lipid compositions used in the capsosome assembly.

\section{CONCLUSION}

We have demonstrated the encapsulation of lysozyme and BDNF within liposomal compartments of capsosomes and the potential of capsosomes in the sustained delivery of proteins. In vitro studies revealed that capsosomes exhibited long-term release kinetics (>2 months) for both lysozyme and BDNF, which can be tuned depending on the lipid 
composition of the liposome compartments. The inclusion of negatively charged lipids resulted in enhanced electrostatic association between the positively charged proteins and the liposomal membrane, resulting in higher protein loading in capsosomes and slower release. These findings highlight the potential of capsosomes in the long-term delivery of protein therapeutics, a promising method that opens new avenues for the treatment of neurodegenerative diseases such as Alzheimer's, Parkinson's disease and sensorineural hearing loss. Future work will focus on developing these capsosomes as delivery carriers for prolonged neurotrophic factors in clinical applications.

\section{SUPPORTING INFORMATION}

Microelectrophoresis measurement of liposome $\zeta$-potential. Fluorescence calibration curves for Lysozyme-FITC and BDNF-FITC. This material is available free of charge via the Internet at http://pubs.acs.org.

\section{AUTHOR INFORMATION}

\section{Corresponding Author}

*Corresponding Author. Email: fcaruso@unimelb.edu.au

\section{Present Addresses}

†Department of Materials, Department of Bioengineering and Institute of Biomedical Engineering, Imperial College London, London SW7 2AZ, UK

$\ddagger$ Department of Chemistry, University of Warwick, Coventry CV4 7AL, UK

§Manufacturing Flagship, CSIRO, Clayton 3168, Australia

\section{Author Contributions}


The manuscript was written through contributions of all authors. All authors have given approval to the final version of the manuscript.

\section{ACKNOWLEDGMENT}

This research was conducted and funded by the Australian Research Council Centre of Excellence in Convergent Bio-Nano Science and Technology (project number CE140100036) and by the Australian Research Council under the Australian Laureate Fellowship (FL120100030) scheme. K. Kempe is grateful to the Alexander von HumboldtFoundation for a Feodor-Lynen Fellowship.

\section{REFERENCES}

(1) Vermonden, T.; Censi, R.; Hennink, W. E. Hydrogels for Protein Delivery. Chem. Rev. 2012, 112, 2853-2888.

(2) Pisal, D. S.; Kosloski, M. P.; Balu-Iyer, S. V. Delivery of Therapeutic Proteins. J. Pharm. Sci. 2010, 99, 2557-2575.

(3) Gupta, S.; Jain, A.; Chakraborty, M.; Sahni, J. K.; Ali, J.; Dang, S. Oral Delivery of Therapeutic Proteins and Peptides: A Review on Recent Developments. Drug Deliv. 2013, 20, 237-246.

(4) Segers, V. F. M.; Lee, R. T. Local Delivery of Proteins and the Use of Self-Assembling Peptides. Drug Discov. Today 2007, 12, 561-568.

(5) Jevševar, S.; Kunstelj, M.; Porekar, V. G. PEGylation of Therapeutic Proteins. Biotechnol. J. 2010, 5, 113-128.

(6) Roberts, M. J.; Bentley, M. D.; Harris, J. M. Chemistry for Peptide and Protein PEGylation. Adv. Drug Deliver. Rev. 2002, 54, 459-476. 
(7) Danhier, F.; Ansorena, E.; Silva, J. M.; Coco, R.; Le Breton, A.; Préat, V. PLGA-Based Nanoparticles: An Overview of Biomedical Applications. J. Control. Release 2012, $161,505-522$.

(8) Gregoriadis, G.; Jain, S.; Papaioannou, I.; Laing, P. Improving the Therapeutic Efficacy of Peptides and Proteins: A Role for Polysialic Acids. Int. J. Pharm. 2005, 300, 125130.

(9) Alcalá-Alcalá, S.; Urbán-Morlán, Z.; Aguilar-Rosas, I.; Quintanar-Guerrero, D. A Biodegradable Polymeric System for Peptide-Protein Delivery Assembled with Porous Microspheres and Nanoparticles, Using an Adsorption/Infiltration Process. Int. J. Nanomed. 2013, 8, 2141-2151.

(10) Vert, M.; Mauduit, J.; Li, S. Biodegradation of PLA/GA Polymers: Increasing Complexity. Biomaterials 1994, 15, 1209-1213.

(11) Swaminathan, J.; Ehrhardt, C. Liposomal Delivery of Proteins and Peptides. Expert Opin. Drug Del. 2012, 9, 1489-1503.

(12) Chang, H.-I.; Yeh, M.-K. Clinical Development of Liposome-Based Drugs: Formulation, Characterization, and Therapeutic Efficacy. Int. J. Nanomed. 2012, 7, 4960.

(13) Allen, T. M.; Cullis, P. R. Liposomal Drug Delivery Systems: From Concept to Clinical Applications. Adv. Drug Deliver. Rev. 2013, 65, 36-48.

(14) Mady, M. M.; Darwish, M. M. Effect of Chitosan Coating on the Characteristics of DPPC Liposomes. J. Adv. Res. 2010, 1, 187-191. 
(15) Amsden, B. Review of Osmotic Pressure Driven Release of Proteins from Monolithic Devices. J. Pharm. Pharm. Sci. 2007, 10, 129-143.

(16) Yeo, Y.; Baek, N.; Park, K. Microencapsulation Methods for Delivery of Protein Drugs. Biotechnol. Bioprocess Eng. 2001, 6, 213-230.

(17) Städler, B.; Chandrawati, R.; Goldie, K.; Caruso, F. Capsosomes: Subcompartmentalizing Polyelectrolyte Capsules Using Liposomes. Langmuir 2009, 25, 6725-6732.

(18) Städler, B.; Chandrawati, R.; Price, A. D.; Chong, S.-F.; Breheney, K.; Postma, A.; Connal, L. A.; Zelikin, A. N.; Caruso, F. A Microreactor with Thousands of Subcompartments: Enzyme-Loaded Liposomes within Polymer Capsules. Angew. Chem., Int. Ed. 2009, 48, 4359-4362.

(19) Chandrawati, R.; Hosta-Rigau, L.; Vanderstraaten, D.; Lokuliyana, S. A.; Städler, B.; Albericio, F.; Caruso, F. Engineering Advanced Capsosomes: Maximizing the Number of Subcompartments, Cargo Retention, and Temperature-Triggered Reaction. ACS Nano 2010, 4, 1351-1361.

(20) Nagahara, A. H.; Tuszynski, M. H. Potential Therapeutic Uses of BDNF in Neurological and Psychiatric Disorders. Nat. Rev. Drug Discov. 2011, 10, 209-219.

(21) Lu, B.; Nagappan, G.; Guan, X.; Nathan, P. J.; Wren, P. BDNF-Based Synaptic Repair as a Disease-Modifying Strategy for Neurodegenerative Diseases. Nat. Rev. Neurosci. 2013, 14, 401-416.

(22) Nagahara, A. H.; Merrill, D. A.; Coppola, G.; Tsukada, S.; Schroeder, B. E.; Shaked, G. M.; Wang, L.; Blesch, A.; Kim, A.; Conner, J. M.; Rockenstein, E.; Chao, M. V.; Koo, E. H.; Geschwind, D.; Masliah, E.; Chiba, A. A.; Tuszynski, M. H. 
Neuroprotective Effects of Brain-Derived Neurotrophic Factor in Rodent and Primate Models of Alzheimer's Disease. Nat. Med. 2009, 15, 331-337.

(23) Fumagalli, F.; Racagni, G.; Riva, M. A. Shedding Light into the Role of BDNF in the Pharmacotherapy of Parkinson's Disease. Pharmacogenomics J. 2006, 6, 95-104.

(24) Wang, Y.; Wise, A. K.; Tan, J.; Maina, J. W.; Shepherd, R. K.; Caruso, F. Small 2014, $10,4244-4248$.

(25) Seeber, S. J.; White, J. L.; Hem, S. L. Predicting the Adsorption of Proteins by Aluminium-Containing Adjuvants. Vaccine 1991, 9, 201-203.

(26) Kuczewski, N.; Porcher, C.; Gaiarsa, J.-L. Activity-Dependent Dendritic Secretion of Brain-Derived Neurotrophic Factor Modulates Synaptic Plasticity. Eur. J. Neurosci. 2010, 32, 1239-1244.

(27) Ono, A.; Takeuchi, K.; Sukenari, A.; Suzuki, T.; Adachi, I.; Ueno, M. Reconsideration of Drug Release from Temperature-Sensitive Liposomes. Biol. Pharm. Bull. 2002, 25 , 97-101.

(28) Anyarambhatla, G. R.; Needham, D. Enhancement of the Phase Transition Permeability of DPPC Liposomes by Incorporation of MPPC: A New Temperature-Sensitive Liposome for Use with Mild Hyperthermia. J. Liposome Res. 1999, 9, 491-506.

(29) Van Slooten, M. L.; Boerman, O.; Romøren, K.; Kedar, E.; Crommelin, D. J. A.; Storm, G. Liposomes as Sustained Release System for Human Interferon- $\Gamma$ : Biopharmaceutical Aspects. BBA-Mol. Cell Biol. L. 2001, 1530, 134-145.

(30) Walker, R. A.; Conboy, J. C.; Richmond, G. L. Molecular Structure and Ordering of Phospholipids at a Liquid-Liquid Interface. Langmuir 1997, 13, 3070-3073. 
(31) Radziejewski, C.; Robinson, R. C.; DiStefano, P. S.; Taylor, J. W. Dimeric Structure and Conformational Stability of Brain-Derived Neurotrophic Factor and Neurotrophin3. Biochemistry 1992, 31, 4431-4436.

(32) Magin, R. L.; Niesman, M. R. Temperature-Dependent Drug Release from Large Unilamellar Liposomes. Canc. Drug. Deliv. 1984, 1, 109-117. 
Table of Contents Graphic

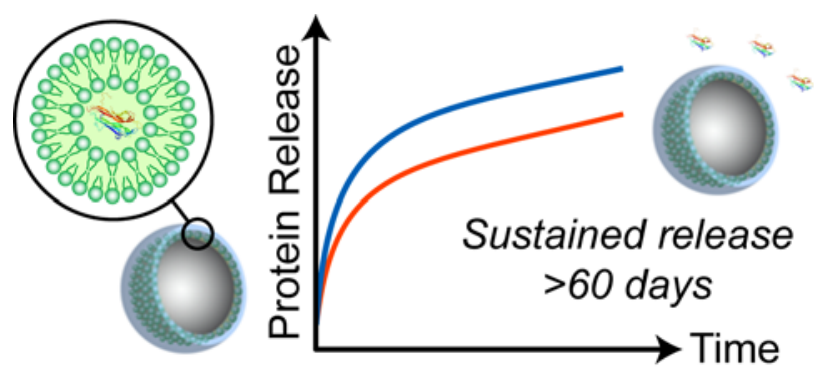




\section{University Library}

\section{- M M N E R VA A gateway to Melbourne's research publications}

Minerva Access is the Institutional Repository of The University of Melbourne

Author/s:

Maina, JW;Richardson, JJ;Chandrawati, R;Kempe, K;van Koeverden, MP;Caruso, F

Title:

Capsosomes as Long-Term Delivery Vehicles for Protein Therapeutics.

Date:

2015-07-09

Citation:

Maina, J. W., Richardson, J. J., Chandrawati, R., Kempe, K., van Koeverden, M. P. \& Caruso, F. (2015). Capsosomes as Long-Term Delivery Vehicles for Protein Therapeutics.. Langmuir: the ACS journal of surfaces and colloids, 31 (28), pp.7776-7781. https://doi.org/10.1021/ acs.langmuir.5b01667.

Persistent Link:

http://hdl.handle.net/11343/90857 